\title{
Diálogos entre Formación Docente en Educación Física y Educación Sexual Integral: acerca de tensiones, discusiones, continuidades y discontinuidades desde la perspectiva de graduados y graduadas en el ISEF No2 Federico W. Dickens, Buenos Aires
}

\author{
Dialogues between Physical Education Teaching Training and Comprehensive Sexual Education Act: tensions, \\ discussions, continuities, and discontinuities from the perspective of undergraduates from ISEF $\mathrm{N}^{\circ} 2$ Federico W. \\ Dickens, Buenos Aires
}

\author{
Sebastián Klein \\ Facultad Latinoamericana de Ciencias Sociales (FLACSO), Argentina \\ sebastianklein_ef@hotmail.com
}

\begin{abstract}
RESUMEN:
El presente artículo de investigación pretende indagar sobre los sentidos y los discursos en torno a la formación docente en la ley de la Educación Sexual Integral en el campo disciplinar de la Educación Física. Se elaboró una investigación del tipo descriptivo bajo el modo de operación cualitativo y diseño de acción-investigación a partir de entrevistas y análisis documental cuyo marco referencial está situado en el Instituto Superior de Educación Física "Federico W. Dickens", Ciudad Autónoma de Buenos Aires, Argentina. A partir del análisis del trabajo de campo, y en articulación con el marco conceptual y el propósito de la investigación, los resultados y sus discusiones están vinculados a la importancia social de que se formen docentes de Educación Física en las perspectivas de la ley en cuestión; en el carácter transversal de sus contenidos y los puntos de contacto con el diseño curricular que opera en las clases de Educación Física; y en la función social de la ley en términos de ampliación de derechos y su interpretación como una herramienta docente. Al finalizar el artículo se propone una serie de interpretaciones a modo de conclusión y posibles líneas de investigación en la temática abordada.
\end{abstract}

Palabras ClaVE: Formación docente, Educación Física, Educación Sexual Integral, Derechos Humanos.

\section{ABSTRACT:}

This research article investigates the senses and the discourses around teaching training in the Comprehensive Sexual Education Act, within the disciplinary field of Physical Education. It presents a descriptive research elaborated with qualitative and action research tools, as it is based on interviews and the analysis of documents. Its referential framework is situated in the Instituto de Formación Superior,"Federico W. Dickens", in Buenos Aires, Argentina. From the analysis of the fieldwork, and in articulation with the conceptual framework and the purpose of the research, the results and their discussions are linked to the social importance of training Physical Education teachers in the perspectives of the above mentioned Act; in the transversal nature of its contents and the contact points with the curriculum that operates in the Physical Education classes; as well as the social function of the Act in terms of extension of rights and its interpretation as a teaching tool. At the end of this article, a series of interpretations are proposed as a conclusion, along with possible lines of research in the subject presented.

KeYwords: Teaching training, Physical Education, Comprehensive Sexual Education Act, Human Rights.

\section{INTRODUCCIÓN}

El presente artículo de investigación pretende indagar sobre los sentidos y los discursos en torno a la formación docente en la ley de la Educación Sexual Integral (en adelante, ESI) en el campo disciplinar de la Educación Física (EF a partir de ahora). Partiendo de este tema de investigación, se elabora un problema central que plantea cuáles son los sentidos y significados atribuidos a la enseñanza de la ESI en la Formación Docente del campo de la EF desde la perspectiva de los/as graduados/as (2018-2020) 
A partir de finales del siglo XX y durante los últimos años, han proliferado en el campo disciplinar de la EF cierta cantidad en ascenso de investigaciones, ponencias, tesis y artículos científicos que vinculan este campo con los estudios de género (Kopelovich y Pansa, 2017). Sin embargo, en el contexto de una cátedra de un profesorado de Educación Física, se dejó entrever que los/as futuros/as docentes desconocían una ley de alcance nacional como la ESI y los lineamientos curriculares para el campo de la EF. A partir de un análisis de las investigaciones o trabajos científicos que se han realizado vinculando la ESI y la EF, se evidenció que son escasos. Más aún en la focalización de la formación docente, situación problemática que motiva la elaboración de este trabajo de investigación. Cabe destacar que la investigación que acá se presenta es una primera aproximación a esta problemática y podría abrir investigaciones y temáticas novedosas en el interior del campo de la EF.

Se elaboró una investigación del tipo descriptivo bajo el modo de operación cualitativo y diseño de acción-investigación a partir de entrevistas y análisis documental cuyo marco referencial está situado en el Instituto Superior de Educación Física "Federico W. Dickens", Ciudad Autónoma de Buenos Aires (CABA en adelante), Argentina. La unidad de análisis está centrado en docentes de EF recientemente graduados/as de dicha institución que hayan atravesado el taller de ESI que se dicta en el profesorado desde el año 2017.

En este apartado introductorio, a continuación se describirá brevemente generalidades, intencionalidad y contexto en torno a la ley de Educación Sexual Integral. Posteriormente, se confeccionará un estado del arte que vincule la formación docente y la enseñanza de la ESI. En el segundo apartado, describiremos las decisiones y estrategias metodológicas de la investigación. En tercer lugar, analizaremos las categorías y clasificaciones interpretadas y puestas en discusión a partir del trabajo de campo atravesadas por el marco teórico seleccionado para cohesionar conceptualmente la investigación. Finalmente, se elaborará una serie de interpretaciones a modo de conclusión y posibles líneas de investigación en la temática abordada.

\subsection{Acerca del Programa Nacional de Educación Sexual Integral: generalidades, intencionalidad política y contexto de producción}

La ley 26.150 fue sancionada el 4 de Octubre de 2006 y promulgada el 23 del mismo mes, bajo la denominación de "Programa Nacional de Educación Sexual Integral". De acuerdo a lo enunciado en sus tres primeros artículos, el programa pretende reivindicar el derecho de los/as educandos/as de recibir educación sexual integral ${ }^{1}$ en centros educativos públicos de gestión estatal y privada, tanto de jurisdicción nacional, provincial y municipal, abarcando todos los niveles del sistema educativo de manera obligatoria. Así, la ley implicaría a nivel nacional que el Estado es garante de asegurar un sistema educativo que abarque las dimensiones de sexualidad integral que plantea el programa. En otras palabras, el programa de la ESI es una ley que supone una ampliación de los derechos de los niños, las niñas y los adolescentes. Si bien no figura en la ley la cuestión del género y de la diversidad cultural, sí es en los lineamientos en que dichos ejes se presentan.

La implementación de la ley fue paulatina y presentó avances y retrocesos en su abordaje. Como veremos más adelante, y partiendo de la premisa que es fundamental la formación en ESI de futuros/as docentes para garantizar el efectivo cumplimiento de la ley, recién en 2017 comienza el dictado del taller del programa en el ISEF N² Federico W. Dickens, luego de más de 10 años de su sanción.

El análisis de las políticas educativas desde enfoques pos-estructuralistas plantea la necesidad de ir más allá del mero estudio del texto de la política, abarcándola desde las dimensiones macro a las micro. Así, se analiza el proyecto contemplando los antecedentes históricos, los temas de agenda política, las influencias de los movimientos sociales, las tensiones con las que se discute y los efectos materiales y simbólicos que impactan en las prácticas sociales. Desde esta perspectiva, entonces, adquiere centralidad el análisis del contexto económico, político, social y cultural de surgimiento de la política educativa en cuestión, el cual, por una cuestión de extensión, no será planteado en el presente artículo. 
Stephen Ball, uno de los investigadores referentes, plantea que las políticas educativas deben ser analizadas no sólo como texto, sino también como discurso (Tello y Mainardes, 2012). Esto último implica considerar las fronteras de pensamiento y acción, es decir, la condición de legitimidad que adquieren determinados agentes sociales, los discursos que se vuelven dominantes - cargados con determinado régimen de verdad - $\mathrm{y}$, por ende, hasta dónde un proyecto de política educativa puede abarcar. Por otro lado, el texto de las políticas educativas es "producto de múltiples influencias como intenciones políticas y negociaciones" que conforma la agenda que disputan los diversos actores sociales, en lo que Ball denominó "ciclo de políticas" (Tello y Mainardes, 2012: 17-18).

En contexto argentino, a principios del siglo XXI se van acrecentando y visibilizando los casos de femicidios que acaecen en el país y más aún los casos de violencia contra la mujer. Como respuesta a esto, proliferan organismos sociales que reclaman derechos de las mujeres e igualdad de género e identidad sexual, colectivos LGTBIIQ+, empoderando voces que históricamente no fueron escuchadas. Masivas manifestaciones callejeras, la lucha por aborto legal, la convocante campaña NiUnaMenos, el Paro Internacional de las mujeres, los ya más de treinta y anualmente más masivos Encuentros de la Mujer. A pesar de la visibilización de estas luchas sociales, aún perduran fuertemente los discursos conservadores que pretenden dar frente a las luchas sociales por la ampliación de derechos.

En este marco, el programa de ESI estuvo fuertemente tensionado por diversos agentes conservadores, fuertemente consolidada en la Iglesia. El consenso y la construcción y sanción de la ley 26.150 implicó varios proyectos hasta llegar al definitivo, a nivel de Congreso como así también en cuanto a las audiencias con otros sectores de la sociedad que representaban diferentes posicionamiento con respecto al programa. El artículo $5^{\circ}$ de la ley es resultado de ello, ya que focaliza sobre la recontextualización de la ley en cada institución y región de acuerdo a la propia idiosincrasia. Así, en este sentido, las disputas y tensiones aún perduran en la actualidad y en la implementación material del programa.

\subsection{Breve repaso del estado del arte: vínculos entre la Formación Docente y la ley de Educación Sexual Integral}

En este apartado se resumirá el estado del arte vinculado a la formación docente focalizada en la ESI en Institutos de Formación Docente y profesorados universitarios en Argentina, particularmente en la Ciudad Autónoma de Buenos Aires, Córdoba y Río Negro. Cabe destacar que, si bien se registran investigaciones que abordan el lugar de la ESI en la formación docente desde diversas perspectivas, no se hallaron en el campo específico de la EF. Uno de los pocos trabajos indagados, que no aborda la ESI pero cuyo núcleo es similar al aquí planteado, lo desarrollaremos más adelante, en el cual se articula la formación docente en EF con la perspectiva de género (Saraví, 1997).

En la investigación que acá presentamos, este apartado estuvo dividido en dos bloques distintos pero interrelacionados. En primer lugar, se abordaron dos producciones científicas que se centran en algunos profesorados universitarios. En este bloque profundizamos en las investigaciones de Jésica Baéz (2020) y Graciela Morgade, Paula Fainsod, Jésica Báez y Eugenia Grotz (2018), ambas desarrolladas en el ámbito de algunas de las carreras de formación docente de la Universidad de Buenos Aires.

En segundo lugar, investigaciones cuyas unidades de análisis están centradas en Institutos de Formación Docente ubicados en Villa María, Córdoba y en Bariloche, Río Negro. En este bloque, abordamos los artículos científicos de Giachero y Sueiro (2018) y de Talani y Viotti (2017) los cuales, como ya hicimos referencia, focalizan en la formación docente en el Programa ESI en institutos terciarios en Córdoba y Río Negro, respectivamente.

En este resumen del estado del arte se registraron investigaciones diferentes entre sí en cuanto a las unidades de análisis, a las poblaciones, a los tipos de instituciones, a los marcos teóricos, y a las latitudes 
del país, pero cuyas conclusiones presentaron continuidades entre sí. En términos generales, podemos afirmar que los resultados de estas investigaciones coinciden en: a)el carácter conflictivo al interior de la institución educativa; b) interpretación estudiantil en torno a la insuficiencia de los contenidos abordados en la formación y sus efectos en la incertidumbre de la futura práctica docente; c) el lugar de la mujer en lo que concierne al lenguaje y/o su visibilización; y d) la falta de transversalidad institucional y fragmentación en cuanto a las "voluntades docentes" de formar desde la perspectiva de la ESI. Más adelante, pondremos a dialogar estos resultados con los interpretados en la investigación que aquí presentamos.

A la vez, este estado de la cuestión nos permite identificar las vacancias y espacios vacíos existentes vinculados con el desarrollo de investigaciones específicas en la formación docente de Educación Física, y plantear la justificación del objeto de investigación que en este artículo se presenta: no se registraron trabajos con rigurosidad científica que tensionen la enseñanza de la ESI en la formación docente en el campo de la EF desde la perspectivas de los/as estudiantes o recientes graduados/as ${ }^{2}$.

\section{Metodología}

Como se anticipó en la introducción de este artículo, el problema central de la investigación que se planteó es: ¿cuáles son los sentidos y significados atribuidos a la enseñanza de la ESI en la Formación Docente del campo de la EF desde la perspectiva de los/as graduados/as (2018-2020)? Esta investigación tuvo desarrollo a partir de un estudio de caso exploratorio (Marradi, Archetti y Piovani, 2007: 242), concretamente radicado en el Instituto Superior de Educación Física No2 Federico W. Dickens (en adelante, ISEF $N^{\circ} 2$ "F.W.D"), ubicado en la Ciudad Autónoma de Buenos Aires. Este instituto de formación docente tiene sede en la zona sur de la ciudad, en el barrio de Parque Chacabuco y es un profesorado de gestión estatal, es decir, depende del Gobierno de la Ciudad de Buenos Aires, área de Educación Superior. Actualmente, cuenta con una matrícula que supera los/as mil estudiantes, distribuidos/asen los tres turnos de cursada (turno mañana, tarde y vespertino), entre cuatro y un curso por año, dependiendo del turno y progresión en la carrera. En tanto marco referencial, cabe destacar dos características de la institución que son relevantes para los resultados de la investigación: por un lado, el turno vespertino estuvo vetado para que las mujeres puedan cursar en esa franja horaria hasta 2018; por otro lado, las asignaturas teóricas-prácticas (esto es, las materias vinculadas con las prácticas deportivas) se dictan mayoritariamente en forma dividida conforme el dato biológico sexual de los/as estudiantes.

Cabe destacar que la unidad de análisis específica fueron los/as graduados/as de dicha institución que culminaron sus estudios entre el 2018 y 2020. El motivo de este recorte estuvo basado en que la inclusión del taller de ESI en el Plan Curricular Institucional fue concretada a fines del año 2017 y se dicta en el $3^{\circ}$ año de la carrera $^{3}$. Por consiguiente, como dicho plan consta de 4 años mínimos de duración de la carrera, son recién los/as egresados/as del 2018 quienes fueron la primer cohorte en tener el taller de ESI en su plan curricular.

Inscripta bajo el modo de operación tendiente hacia lo cualitativo (Sirvent, 2005), esta investigación fue del tipo descriptivo de acuerdo a Sampieri (2010) ya que se pretendió comprender y describir un fenómeno social desde la perspectiva de los actores que se insertan en el mismo.

En cuanto al diseño de investigación, la cual organizó metodológicamente las decisiones del proyecto (Marradi et al, 2007: 71-85), se optó por el modelo de investigación-acción a partir de dos decisiones asumidas: por un lado, debido a la intencionalidad de construir conocimiento científico en la articulación investigador-sujetos de la investigación en su contexto social específico (Sirvent, 2009; Sampieri, 2010); por el otro lado, la concepción de la investigación como acción que influye en las prácticas y dinámicas institucionales con la finalidad de transformar la realidad en la formación docente desde la perspectiva de la ESI (Sampieri, 2010). Con respecto a la primera cuestión, la construcción de conocimiento científico no vino pre-establecido a priori por parte del investigador, sino que por el contrario los sujetos de la investigación tuvieron un lugar protagónico en el proceso. En cuanto a la influencia, como se argumentará en 
las conclusiones, la investigación posibilitó visibilizar problemáticas institucionales y sistémicas en cuanto a la formación en ESI de los/as fututos/as docentes; asimismo, permitió sistematizar y estructurar las diversas experiencias, expectativas, sentidos y prácticas de los/as agentes educativos involucrados/as.

En cuanto a las estrategias e instrumentos de recolección de la evidencia empírica, se decidió aplicar para la confección de esta investigación entrevistas virtuales semi-estructuradas ${ }^{4}$ (Marradi, 2007: 217) y análisis documental. Como se anticipó en los párrafos anteriores, las entrevistas se realizaron a un total de 6 egresados/as entre los años 2018 y 2020 del ISEF N² F.W.D, específicamente 3 varones y 3 mujeres. Por el otro lado, los documentos analizados fueron la ley y los lineamientos curriculares enmarcados en el Programa Nacional de ESI en tanto fuentes primarias.

\section{Resultados y Discusión}

A partir del análisis, la clasificación y la codificación del material empírico, con la articulación del marco conceptual y del propósito de la investigación, en este apartado desarrollaremos los resultados de la investigación. A continuación se expondrán las discusiones emergentes en relación a: la importancia de la formación de docentes de Educación Física en las perspectivas de la ley en cuestión; el carácter transversal de sus contenidos; los puntos de contacto con el diseño curricular que opera en las clases de Educación Física; y la función social de la ley en términos de ampliación de derechos y su interpretación como una herramienta docente.

\subsection{Entre lo instituido y lo transversal: tradición, disputa y tensiones que conviven en la formación del docente de Educación Física}

Los discursos de los egresados y las egresadas señalan la importancia "clave" que tiene la enseñanza de la ESI en la formación docente del/a futuro/a profesor/a de Educación Física. Se analizó y registró esta afirmación representada por el total de entrevistados/as a partir de dos clasificaciones generales: el fundamento de la "importancia" radica, por un lado, en que la ESI irrumpe habilitando discusiones al interior del instituto; por otro lado, esta irrupción pretende ser disruptiva porque "viene a romper con lo instituido" en el campo disciplinar de la Educación Física.

En cuanto a la primer clasificación, la mayoría de los/as entrevistados/as sostienen que esta habilitación en las discusiones al interior del profesorado se origina a partir de la interpelación del taller de ESI sobre los/as estudiantes que motorizan las discusiones “a otros espacios institucionales”, como por ejemplo la Jornada ESI. En este sentido, afirma uno de los entrevistados: "Si no hubiera sido por el centro de estudiantes, no me habría interesado en la temática. En ese sentido, me formó más ese espacio que la institución en sí” (Entrevistado No 3, Profesor Superior de Educación Física egresado en 2020).

$\mathrm{Al}$ respecto, Blanco elaboró un trabajo de investigación en relación a la inclusión en la agenda política institucional, efecto de las demandas provenientes de movimientos feministas y de la diversidad sexual, las reivindicaciones y luchas sociales en torno al género, las mujeres y los colectivos LGBTIIQ+ en las agrupaciones estudiantiles en la formación superior (Blanco, 2014). La disputa de género al interior del ISEF N² "F.W.D" fue motorizada ampliamente por el sector estudiantil y respaldada, en gran medida, por el paradigma educativo-pedagógico que sostienen algunos docentes en el profesorado en relación al campo de la EF. En torno a las estrategias de las agrupaciones estudiantiles que describe Blanco en su investigación interesa, conforme el hilo conductor que venimos desarrollando, la última: "interpelar a partir de cuestiones de la vida cotidiana de la comunidad estudiantil, especialmente en torno a las condiciones en las que cursan la carrera”. Esto es, precisamente, lo que fue expresado en más de la mitad de los/as entrevistados/ as, más que nada las graduadas mujeres: "medidas" de visibilización de la violencia machista y excluyente 
en que se desarrollan ciertas prácticas. Entre otros proyectos impulsados por los/as estudiantes, destacaron: "empapelado del profesorado con frases misóginas y violentas en términos de género de frases de docentes en relación a la inferioridad de la mujer o del colectivo de gays o lesbianas", "coordinación de las jornadas de ESI con algunos/as docentes", "clínicas de deportes mixtos".

La totalidad de los/as egresados/as entrevistados/as se pronunció, de alguna u otra manera, acerca de cómo el taller de ESI problematizó en torno a la jerarquización masculina sobre la femenina a la hora de pensar las prácticas deportivas al interior del profesorado. A partir de ello, se explicitaron los argumentos "biologicistas" y "machistas" por los cuales se justificaba que las estudiantes mujeres no podían cursar en el Turno Vespertino ni practicar deportes junto a los estudiantes varones. Desde la interpretación de los/as entrevistados/as, algunos directivos de aquella época y algunos docentes sostenían que el turno vespertino era un horario "peligroso" para las estudiantes mujeres y que por ello "no se anotaban" para cursar en esa franja. Sin embargo, los datos que elaboró el centro de estudiantes sostenía que "no se anotaban porque no les daban la oportunidad", datos que "se constataron con la realidad" cuando "se permitió institucionalmente que se anotaran a cualquiera de los turnos" y se materializó que haya mujeres cursando en ese turno finalmente en 2018 (Entrevistada N ${ }^{\circ}$ 6, Profesor Superior de Educación Física, egresada en 2019).Por el otro lado, los discursos que denegaron -y siguen negando- las prácticas deportivas mixtas giran en torno a argumentos "biologicistas" y "neurocientíficos". Entre ellos, sostenían que las mujeres y los varones tienen características diferentes fisiológica y hormonalmente y que esta condición puede representar un peligro para las mujeres por la fuerza que podrían llegar a ejercer los varones. Asimismo, en términos de competitividad, los varones llegan a un nivel de rendimiento deportivo que las mujeres no.

La mitad de los/as entrevistados/as profundizó sobre estas consideraciones en torno a la necesidad de pensar el profesorado como lo que es: "un instituto de formación docente y no un club deportivo" (Entrevistada $\mathrm{N}^{\circ} 6$ ). En ese sentido, sostuvieron que "venimos a formarnos como docentes y no como deportistas" y "tenemos que aprender a enseñar y no a competir profesionalmente" (Entrevistada $\mathrm{N}^{\circ}$ 6). Asimismo, surgió la noción de que dentro de la formación docente se originan desiguales distribuciones de los contenidos enseñados en términos de prácticas deportivas que se enseñan para mujeres o para varones excluyentemente. Sentencia una de las entrevistadas: "Por una formación docente desigual en términos de género, yo no tengo herramientas para enseñar rugby a un grupo de alumnas, por ejemplo, y eso perpetúa las desigualdades educativas y vulnera derechos humanos, como el acceso al deporte" (Entrevistada $\mathrm{N}^{\circ} 6$ )

Esta diferencia que marcan los/as egresados/as tiene larga data. En el libro producto de su tesis de maestría, Ángela Aisenstein ya cuestionaba que, si bien una institución de formación docente no es una entidad deportiva sino educativa, hay elementos teórico-empíricos que dan cuenta que en el caso del profesorado de EF se presentan varias similitudes entre ambas (Aisenstein, 1995; citado en Saraví, 1997). Sin embargo, entre los discursos de los entrevistados también se encuentra la preocupación en torno la concepción de la "biología como el destino educativo" 5 en el campo de la formación docente y quela reproducción de esos modelos binarios y excluyentes en la formación docente, fundamentados a partir de perspectivas biologicistas, podría consolidar la desigualdad social y educativa de las mujeres y los colectivos disidentes en torno a las prácticas corporales en la escuela. Al respecto, ya Jorge Saraví señalaba en 1997:

Existe también el de los esquemas, prejuicios y estereotipos originados en las consignas, pautas, formas de trabajo y actitudes, explícitos o implícitos, que reciben y viven cotidianamente los/as estudiantes de educación física en formación con relación al tema del género y que se transmitirían y repercutirían posteriormente en el ejercicio profesional (Saraví, 1997: 33)

Los/as egresados/as entrevistados/as afirman que el taller de ESI, a pesar que no les dio herramientas suficientes para la implementación de sus lineamientos a sus prácticas docentes, como veremos más adelante, sí les proporcionó bibliografía y marcos legales para desandar los argumentos biologicistas anteriormente desarrollados en que en términos institucionales se argumentó que los deportes enseñados no sean mixtos 
ni que las mujeres cursen en el turno vespertino (Entrevistada $N^{\circ} 5$, Profesora Superior de Educación Física, egresada en 2018).

En cuanto a la segunda clasificación, los/as entrevistados/as afirmaron, de una u otra manera, que la ESI "vino a romper con lo instituido" o "lo instaurado" en la "tradición" de la EF. Se analizó y registró el material empírico en esta línea a partir de tres ejes centrales: en torno a la concepción de cuerpo, de deporte y en torno a las clases de EF -que será profundizado en el último apartado de este capítulo-. En cuanto a lo corporal, se hizo referencia a cierta tradición hegemónica conservadora en que se piensa los cuerpos en la EF, más que nada vinculado históricamente a lo "elitista" y "militarista”, y puntualizaron sobre el aporte de la ESI para replantear la concepción de los cuerpos. En esta última línea, uno de los entrevistados, sostuvo que "abordando la ESI también se discute que un hombre puede tener otro tipo de elegancia, de conducta, de gesto corporal que no se relaciona con el modelo hegemónico" (Entrevistado $\mathrm{N}^{\circ} 1$ ). Otra ejemplificación textual en relación a los discursos de los entrevistados que da cuenta de la problematización de la ESI acerca de la perspectiva "hegemónica" de lo corporal en el campo de la EF se centró en la disputa en torno a los parámetros del cuerpo "ideal":

La ESI puede romper mucho con estos esquemas (...) con los estereotipos y las construcciones hegemónicas de belleza y es algo que la EF reafirma todo el tiempo, esto de que el cuerpo esbelto y atlético es el mejor y al gordito lo cargan o va al arco. Por eso es importante una buena formación en ESI, nos ayudaría un montón a repensar los problemas cotidianos (Entrevistado $\mathrm{N}^{\circ} 4$ )

En los lineamientos curriculares de la ESI (2008), otro de los documentos oficiales analizados, se ofrece una guía de temáticas, contenidos y perspectivas que se pueden transversalizar en torno a los intereses y enfoques generales de la ESI en los diferentes niveles educativos y asignaturas escolares. Para el caso de la EF escolar, se puntualiza sobre la construcción hegemónica del cuerpo ideal, la belleza, los medios de comunicación y el consumo, como surge a partir del análisis de las entrevistas, abordándolos ya desde el nivel primario. Para el nivel primario: "La reflexión acerca de los modelos corporales presentes en los medios de comunicación, en la publicidad y en el deporte espectáculo" (2008: 25). Para el caso del nivel secundario: "La indagación y análisis crítico sobre la construcción social e histórica del ideal de la belleza y del cuerpo para varones y mujeres" y lo relativo a "cuestionar la valoración de patrones hegemónicos de belleza y la relación con el consumo" (2008: 48).

Está claro que no es en el campo exclusivo de la EF en el cual se construyen los cuerpos bajo los parámetros normativos de la ciencia androcentrista en general ni por los determinismos biológicos en particular. Al respecto, en una investigación que pretende transversalizar la perspectiva de género en torno a la configuración de los cuerpos sexuados en dos asignaturas de nivel medio, Morgade, Fainsod, Del Cerro y Busca señalan que:

La enseñanza acerca de los cuerpos sexuados estuvo dominada por el modelo biomédico que sostiene su autoridad epistémica sobre "la verdad de los cuerpos" anclada en el orden natural y legitimado por su objetividad científica (...)Este papel determinante de lo biológico se enlaza a una conceptualización androcéntrica de la ciencia y al esencialismo presente en los modos hegemónicos de caracterizar las formas de habitar los cuerpos, las identidades de género y las sexualidades. El "modelo biomédico hegemónico" legitima y pone en práctica estos "núcleos duros", clasificando los cuerpos en un continuo de mayor o menor distancia a aquel orden natural. (2016: 165)

Aunque excede la intencionalidad del presente artículo introductorio a la investigación, es pertinente al menos enunciar algunos lineamientos conceptuales con os que comprenderemos al cuerpo. Sin negar la existencia de sistemas anatómicos-fisiológicos, el cuerpo no está determinado a lo orgánico. El cuerpo y los usos o las prácticas corporales no tienen nada de natural (Scharagrodsky, 2006; Scharagrodsky, 2007; Crisorio, 2009; Crisorio, 2015). La investigación aquí presentada se fundamenta en los aportes de Foucault (2002; 2006) para interpretar la dimensión política del cuerpo; en Butler (2012) para dar cuenta del carácter performativo del discurso en la materialidad del cuerpo; y en Crisorio (2015) para evitar la reducción de las "prácticas corporales" meramente a "actividad física”. 
En cuanto a la dimensión de lo deportivo, también hay consenso entre las personas entrevistadas cuando plantean la tensión que existe entre las perspectivas competitivas del deporte que domina la escena en, por ejemplo, la formación docente, y el enfoque "inclusivo" o de respeto por las "otredades" que se desprende de los lineamientos en torno a la ley que aquí tematizamos. En esta línea pareciera que la ESI tensiona, en el campo de la EF, entendido en sentido bourdieano, diferentes enfoques y perspectivas que disputan hegemonía. En palabas de uno de los/as entrevistados/as: "la ESI viene como a hacerle fuerza a lo que está instituido, que en el profesorado y en la EF es el deporte competitivo, que viene de hace miles de años y son todos deportes occidentales con fuerte sesgo sexista" (Entrevistado $\mathrm{N}^{\circ}$ 4). A esto se le suman, las contradicciones que despierta la ESI dentro de la EF, al menos en la CABA, en donde las clases no son mixtas en el sistema educativo de nivel medio. Como argumentan los/as egresados/as, la ESI "pelea sola contra la marea": el respeto por el otro y la otra que pregona, incluso en la formación docente de EF, se ve confrontada con la misma idiosincrasia del profesorado y con el sistema educativo mismo. En este punto se torna interesante plantear la matriz disciplinar de la EF en términos de continuidad histórica. Como sostiene Aisenstein (2006; 2015), a pesar de múltiples mutaciones en el devenir histórico en cuanto a los pedagógico, didáctico y curricular, incluida la consolidación del deporte como contenido a enseñar, la matriz disciplinar de la EF tendió históricamente a constituir la "continuidad de la estrategia reguladora sobre los cuerpos en la escuela y por fuera de ella" procurando al mismo tiempo "mantener y acentuar las diferencias sexuales" (2015: 152).

Hasta aquí, los argumentos de los/as entrevistados/as sostienen que los discursos de la ESI son disruptivos y ponen en tensión la tradición de la formación docente y el mismo campo disciplinar. En consonancia con lo anterior, se registró en que la totalidad de los enunciados de los agentes entrevistados se evidencia como categoría central de análisis la cuestión de la transversalidad de los contenidos de la ESI en relación al resto de las asignaturas que conforman el Plan Curricular del instituto. Los/as entrevistados/as sostuvieron que son pocas las otras asignaturas que retoman las nociones fundamentales de la ley de ESI, que queda a criterio de cada docente su inclusión o no en los contenidos de su asignatura a cargo ${ }^{6}$. En líneas generales, todos/ as estuvieron de acuerdo al afirmar que fue más por iniciativa de los/as estudiantes que la ESI haya estado instalada en la agenda educativa de la institución, que por los/as directivos/as o los/as docentes mismos.

La cuestión de la transversalidad fue abordada en el estado del arte de este artículo y se relaciona con investigaciones centradas en la formación docente y es, al menos a priori, una continuidad que se repite en diferentes carreras formativas. En esta línea, Baez (2020) concluye en su investigación que en la formación docente de la ESI la transversalidad no se efectiviza, y da cuenta de una "secuencia fragmentada de voluntades docentes marginales", que, como se sostuvo en el párrafo anterior, responde al criterio de cada uno/a de los/ as docentes y su voluntad de transversalizar sus contenidos en función de los planteos de la ESI. Por otro lado, Morgade et al (2018) señalan la fragmentación en la formación docente vinculada con la falta de criterios unificados para materializar la ley en el plan institucional transversal entre las asignaturas que lo integran. Por otro lado, la cuestión de la transversalidad también figura fuertemente en los lineamientos curriculares. Para la problemática que estamos abordando en estas líneas, el documento sentencia que: "En caso de que se defina el tratamiento de la ESI en el marco de un espacio curricular específico, el abordaje deberá ser integral para evitar reduccionismos que podrían empobrecer el enfoque interdisciplinario que aquí se sustenta" (2008: 16).

El fenómeno que se dicte "ESI sólo en el taller de ESI" (Entrevistada $\mathrm{N}^{\circ} 6$ ) supone que el conjunto de la institución no se inclina por las premisas que instaura el programa con fuerza de ley, sino que apenas es sostenida por algunas individualidades. Sin embargo, los/as egresados/as van un poco más allá: que haya docentes que no den contenido de ESI transversalmente con los contenidos de la asignatura en cuestión es problemático, pero no tanto como aquellos discursos docentes que siguen sosteniendo que el varón es más "fuerte" o "valioso" que las mujeres, o que las mujeres son más "estéticas" o "elegantes" que los varones.

Por otro lado, también hicieron mención en relación a los ejes nodales de cada asignatura: no interpretan tan "grave" que no se aborde ESI en "Anatomía" como sí creen que es "gravísimo" que no se articulen los 
lineamientos de la ESI con las "Didácticas" o con el "Campo de la Formación Profesional". Efectivamente, ven aquí una grave contradicción, porque la ley de la ESI sostiene que los docentes deben formarse en sus lineamientos curriculares pero los/as docentes de las prácticas profesionales de los/as estudiantes no los tienen en consideración.

A partir del análisis precedente, sostenemos que los/as entrevistados/as consideran que sin transversalidad no hay formación docente de calidad. En torno a esto, sostuvieron que la formación docente recibida en ESI fue insuficiente y no les proporcionó las "herramientas" necesarias para garantizarla en sus clases de EF. Por consiguiente, todos/as sostuvieron que necesitan una formación más específica e intensa para "aplicar" los lineamientos curriculares a su práctica docente eficientemente, a pesar de resaltar que sí aprendieron conceptualmente algunas nociones relevantes. Sobre esto último, remarcaron su importancia porque hay diversos agentes educativos que están en contra de la implementación de la ESI y que la fundamentación conceptual y jurídica podría defenderla ante las autoridades o directivos escolares.

\subsection{La Educación Sexual Integral como derecho en las escuelas. Acerca de su abordaje en las clases de Educación Física}

Este apartado describirá los análisis realizados en función del lugar de la ESI en el ámbito escolar y se hará breve mención a la incidencia de la formación docente en la práctica del/la docente de EF. Esta descripción estará dividida en dos grandes grupos: por un lado, la concepción de derecho que implica la sanción de la ley de ESI; por otro lado, la conceptualización de la ESI como una "herramienta" en el discurso de los/as docentes.

Como se mencionó, un primer grupo de clasificación está vinculado con la noción de que "la ley de la ESI vino a garantizar y ampliar derechos" en la escuela (Entrevistada $\mathrm{N}^{\circ}$ 6). La totalidad de entrevistados/as sostuvo que la aplicación efectiva de la ESI y los lineamientos curriculares propuestos visibiliza problemáticas históricas y las organiza en el marco de la ley. En este sentido, una de las entrevistadas sostuvo que "en la escuela, siempre existieron personas que no se auto-percibían con el sexo asignado al nacer y vivieron disimulando, aparentando; la ESI promueve que nunca más" (Entrevistada N², Profesora Superior de Educación Física, egresada en 2019).

Los sentidos y representaciones en torno de la ESI, si bien se vinculan en parte con la educación reproductiva, le asignan un carácter "integral” con respecto al paradigma sexual tradicional, como ya hicimos referencia. En esta línea, se pudo entrever los diferentes discursos y sentidos que opera en los/as entrevistados/ as, que se orientaron hacia la detección de problemáticas o violencias, tematizaron las desigualdades poniendo la lupa sobre los discursos discriminatorios o de odio y se estructuraron a partir de la dignidad humana para que "tengan voz quienes nunca la tuvieron" y "puedan ser llamados como lo deseen”. Es en esta línea que los lineamientos curriculares de la ESI se plantean como propósito formativo:

Promover aprendizajes de competencias relacionadas con la prevención de las diversas formas de vulneración de derechos: maltrato infantil, abuso sexual, trata de niños (...) Propiciar aprendizajes basados en el respeto por la diversidad y el rechazo por todas las formas de discriminación. (2008: 14)

En líneas generales, podemos afirmar que el análisis precedente sobre los sentidos en torno a la ESI de los/ as docentes recientemente graduados/as pone de manifiesto la pretensión de la ESI de "reformular" el vínculo social en la escuela desde los ejes que estructuran el programa ESI. El segundo grupo de clasificación de las categorías es precisamente la noción de la ESI como "herramienta" de "la docencia" en general y también del/ a profesor/a de EF en particular.

En efecto, la totalidad de la muestra hizo referencia a la ESI como una "herramienta docente para todos los niveles" en, al menos, dos sentidos de acuerdo a lo analizado. En primer lugar, aparece como un "arma" o un instrumento de trabajo para ser utilizado en sus clases escolares o encuentros educativos. Más allá del carácter instrumental que le asignan, los/as entrevistados/as resaltaron más la cuestión de que deben replicarlo en 
todos los niveles educativos. Este "deber" se relaciona con la obligatoriedad que organiza la fuerza de ley con la que la ESI fue sancionada. "Responsabilidad”, "obligación”, "compromiso docente”, entre otras, son algunas de las expresiones textuales utilizadas por los agentes educativos consultados en este punto. Más concretamente:

Yo creo que la ESI es como un arma de trabajo, ¿̨no? Como una herramienta de trabajo (...) En mi formación docente el taller fue súper importante. Hay una institución formativa que te dice que es un contenido que tenés que abordar, es diferente a decir que es una mirada o forma de ver...No es una opción, acá hay una ley que dice qué tenés que abordar, es como responsabilidad docente, ciudadana (Entrevistado $\mathrm{N}^{\circ} 1$ ).

En segundo término, los sentidos en torno a la concepción de la ESI como "herramienta" se asociaron con el rol clave que asume la formación docente. Puntualmente, los planteos se presentaron en torno al interrogante si la ESI por ley debe ser aplicada desde nivel inicial al superior, por qué motivo en la formación docente de EF el taller de ESI recién está en el tercer año, cuando la didáctica y prácticas de nivel inicial son en segundo. En otras palabras, si la ESI es un derecho para todas las infancias escolarizadas de todos los niveles, ¿̇cómo es posible que en la formación docente haya contenidos teóricos y prácticas profesionales en niveles educativos sin contemplar una ley como la ESI?

En el campo escolar, los discursos y sentidos de los/as entrevistados/as se complementan bajo la premisa de que los contenidos de la EF son idóneos para abordar la ESI. Entre sus enunciados subyace la comparación con otras asignaturas escolares, como la matemática, sobre la cual está instalado el preconcepto que debe "forzar" su relación con los lineamientos de la ESI. En cambio, la EF escolar está "muy vinculada” con el marco pedagógico de la ESI: esta relación se da en el "contexto natural de una clase de EF (...) La EF te da el contexto sólo para abordar la ESI” (Entrevistado N ${ }^{\circ}$ ).

Los contenidos y las prácticas corporales en que se estructura la EF escolar se organizan en relación a otros y otras. En esta línea, casi todos/as los/as entrevistados/as hicieron referencia al abordaje desde el marco de la ESI de los vínculos sociales a partir de la enseñanza de la EF en la escuela. Las prácticas lúdicas, motoras y deportivas a las que se referenciaron se dan en el contexto de interacción con otros/as donde se ponen en juego los lazos sociales para respetar, valorar y aceptar al otro en tanto otro. Al respecto, una de las entrevistadas sostuvo que "el hecho de jugar con un compañero o compañera que sea del sexo distinto al mío ayuda a la grupalidad, al compañerismo, a la confianza con el otro y la otra, me parece súper importante" (Entrevistada $\mathrm{N}^{\circ}$ 2). En la configuración de estos vínculos entre pares, la ESI pretendería "quitarnos los rótulos" en torno a los estereotipos corporales impuestos socialmente y abordar las situaciones de discriminación y burlas en torno a aquellos, focalizado en la dimensión de la construcción de una masculinidad y una feminidad valida por sobre otras formas posibles de "ser varón" y "ser mujer".

Conforme los discursos de los/as entrevistados/as, y a partir de los lineamientos de la ESI, en las clases de EF se pueden poner en circulación "vivencias" de los/as estudiantes en torno al cuerpo, al deporte, y a los juegos. En primer término, la totalidad de la muestra hizo mención al "cuidado del cuerpo y del/a otro/a" en tanto eje de la ESI, en diálogo con los contenidos de la EF a partir de la noción del "propio cuerpo en relación con los otros" prescripto curricularmente. En líneas generales, los argumentos en torno a la noción de que cuidar el cuerpo de uno/a y cuidar al del/a otro/a se puede abordar con facilidad en las clases de EF, interesó particularmente el siguiente enunciado textual: "cuidar mi cuerpo y el del otro conlleva la comprensión de que mi cuerpo es mío, yo decido sobre él y de que es inviolentable” (Entrevistado $\mathrm{N}^{\circ} 3$, Profesor Superior de Educación Física, egresado en 2020).En cuanto al planteo de los lineamientos de la ESI en cuanto al lugar de la educación del cuerpo en el contexto de la EF escolar, señala que el cuerpo y el movimiento en interacción con otros/as "constituyen dimensiones significativas en la construcción de la identidad" (2008: 24). A partir de esta noción, se proponen guías en torno al abordaje de prácticas corporales "en condiciones de igualdad", "enfatizando respeto, cuidado, aceptación y valoración de la diversidad", considerando la "responsabilidad, solidaridad, respeto y cuidado de sí mismo y de los otros/as a través de actividades motrices que posibiliten la resolución de conflictos cotidianos y la convivencia democrática", entre otros diversos postulados. 
En términos históricos, la EF escolar estuvo signada por la intencionalidad política de controlar y moldear los cuerpos en condiciones desiguales conforme el dato biológico de la genitalidad. Como afirma Scharagrodsky "los objetivos de estas prácticas corporales avalaron y legitimaron la construcción de estereotipos sociales y sexuales: desarrollar la fuerza en el varón y la coordinación y el ritmo en las mujeres" (2008: 11). En relación a la configuración de los cuerpos en las escuelas actualmente, en función de los discursos de los/as egresados/as, aún existen mecanismos y dispositivos de regulación de los cuerpos más sutiles, aunque diferentes, pero no por ello menos eficientes. Se evidencian situaciones de violencia sobre los cuerpos desde múltiples posicionamientos en las cuales se consolidan relaciones desiguales de poder a partir de la instauración del "deber ser" corporal para cada género, configurando un orden sexual jerarquizado. Asimismo, estas relaciones de poder se registran en la desigual distribución de los cuerpos en el tiempo o espacio escolar o en enunciados denigrantes en torno a los usos del cuerpo, ejemplificado a partir de los discursos de los/as entrevistados/as en burlas o actos de discriminación. En otras palabras, cuando" se conservan ciertas relaciones de dominación, ciertos derechos y ciertos privilegios cuyo punto de ataque primario es el cuerpo y su fin la esencialización del mismo” (2008: 12). A pesar de esto, el marco conceptual de la presente investigación reniega del determinismo de posiciones estructuralistas sin fisuras que postulan las teorías reproductivistas en el campo de la educación. Por el contrario, como en todo orden de lo social, existen resistencias de los agentes educativos y clivajes de fisura por los cuales se pueden alterar los modelos que construyen hegemonía. Podemos incluir la ESI en esa clave y coincidimos con Morgade y su equipo de investigación cuando señalan su "convencimiento del lugar insoslayable que ocupa la escuela en la construcción de nuevas posibilidades y horizontes en la construcción de los cuerpos sexuados" (Morgade et al, 2016).

Con respecto a los enfoques de las experiencias escolares vinculadas a lo deportivo, hubo predominio de la temática de la mixidad en las clases de EF en nivel secundario en comparación con los efectos pedagógicos enriquecedores de trabajar con pares de diferentes sexos en el nivel primario. Asimismo, los significantes más repetidos por los/as entrevistados/as en torno al deporte fueron "competencia", "exclusión" y "respeto". Por otro lado, los discursos giraron en torno al juego como una herramienta didáctica o un "medio para" trabajar los diferentes lineamientos curriculares, organizados en los cinco ejes de la ESI, en los patios o canchas escolares.

Es pertinente profundizar estos enunciados con bibliografía conceptual que enmarca esta investigación. En cuanto al carácter de exclusión del campo deportivo en la escuela, está instalada la percepción de la mixidad como la contracara para incluir a quienes quedan "por fuera" del mismo en términos de rendimiento. En la misma línea en que se dirigen los discursos en torno al juego como recurso didáctico, podemos interpretar que la finalidad última de estos enunciados tiene que ver con priorizar el rendimiento o la adquisición de alguna habilidad, más que el placer del juego y goce de compartir con el/la otro/a. En el ya clásico "Juntos pero no revueltos", Scharagrodsky plantea principalmente que el hecho de "estar juntos/as" en la práctica deportiva o escolar no garantiza igualdad de condiciones entre varones, mujeres y disidencias, ya que de todos modos aún persisten y perduran relaciones desiguales en términos de jerarquías y ejercicios de poder (Scharagrodsky, 2004). En otro texto pero en la misma línea, expresa que "las prácticas deportivas tienen- y han tenido - aún un papel central en el modelado de diversas masculinidades, especialmente hegemónicas y subordinadas" (Connell, 1995; citado en Scharagrodsky, 2007: 10). Graciela Morgade también se expresa en relación al lugar del deporte escolar - aunque señala los contenidos de EF en general, no simplemente circunscripto al campo deportivo- en la conformación de "una forma de masculinidad hegemónica", efecto del ideal de alto rendimiento de la matriz disciplinar de la EF que aún disputa territorio en el patio escolar, y "que desalienta y en ocasiones deja afuera a un importante número de chicos y chicas de sus clases" (2015: 177). Cabe destacar también los aportes de Saraví (1997), quien coincide con los planteos de ambos autores/ as precedentes en relación las condiciones de discriminación, segregación, dominación y exclusión en cuanto a los roles de género en clave heteronormativa que perdura en el campo de la EF. Saraví puntualiza en las frases 
discriminatorias en los patios y centraliza su preocupación en la formación docente de EF en perspectiva de género, reafirmando la convicción de que los/as docentes en formación se deben ser interpelados en su trayecto educativo desde aquellos posicionamientos. Hace ya 24 años que se planteaba la siguiente pregunta: "¿no podrían ser las cuestiones de género un contenido para el futuro/a docente de educación física en formación?” (Saraví, 1997: 32). Asimismo, si bien la coincide en que las clases mixtas no alterarían la composición del ejercicio desigual de poder entre los géneros, sostiene que el deporte separado por sexo está constituido como uno de los edificios más visibles del sistema patriarcal y que por tal debe ser demolido.

A pesar de estas últimas consideraciones, ya hicimos mención a la "falta de herramientas" o las complejidades para "aplicar" estos puntos de contactos de la ESI a las clases de EF. En efecto, la totalidad de la muestra encontró obstáculos para atravesar los lineamientos de la ESI con el diseño curricular de la EF, lo cual centró la mirada en la formación docente. Sin embargo, hay algo que se repite en los discursos de los/as entrevistados/as, que podríamos resumir así: sin formación docente en ESI, las prácticas de la EF escolar serían aún más desiguales. Desde su percepción, si no se formara a los/as futuros/as docentes de EF en la perspectiva de la ESI, las prácticas docentes del área curricular de la EF difícilmente se modifiquen en dirección de la ampliación de derechos. En palabras de una de los/as entrevistados/as:

(Sin formación en ESI) se seguirá reproduciendo lo que vivimos en la escuela. Se seguirá burlando por el físico del otro, se seguirá limitando el desarrollo motriz conforme las prácticas educativas diferenciadas por sexo. Los pibes y las pibas de la escuela sí lo demandan ahora (Entrevistada $\mathrm{N}^{\circ} 5$ )

\section{Conclusiones}

En este artículo se presentó un problema de investigación vinculado alos sentidos y significados atribuidos a la enseñanza de la ESI en la Formación Docente del campo de la EF desde la perspectiva de los/as graduados/as (2018-2020). Se abordó una introducción a la ley en relación a sus características generales, puntos estructurales, contexto de producción y al estado de la cuestión vinculado con las investigaciones realizadas sobre la temática. Asimismo, se describieron las decisiones metodológicas en torno al proceso de confrontación teórico-empírico para llevar adelante la investigación, inscripta en las lógicas cualitativas. En cuanto a la interpretación de los resultados planteados a partir del material empírico y las discusiones con el marco conceptual, se indagó principalmente la importancia de la formación en ESI atravesada por perspectiva de género en la carrera de los/as futuros/as docentes de EF en una institución de CABA. Esta indagación derivó en temáticas que estuvieron vinculadas con la tradicional matriz disciplinar de la EF y las tensiones y disputas que implica la perspectiva teórica y pedagógica de la ESI en la formación docente del profesorado en cuestión, la transversalidad, las problemáticas en la formación y los recursos didácticos. Asimismo, conforme el modelo de investigación-acción, la investigación posibilitó visibilizar problemáticas y sistematizar experiencias, prácticas y sentidos en torno a la implementación del taller de la ESI en la formación docente en general e institucional en particular. En esta línea, cobra relevancia la potencia del modelo: la posibilidad de socializar para transformar aquello que deba ser transformado y, en definitiva, ampliar y garantizar derechos.

De manera preliminar, aunque no concluyente, podemos realizar una lectura en clave de género de los discursos esgrimidos por los/as graduados/as. Los tres entrevistados, aunque con matices, problematizaron más que nada la formación docente y los efectos en la práctica profesional. Por otro lado, podemos interpretar que las tres entrevistadas, además de aquello, pusieron foco también en las desigualdades en perspectiva de género que como mujeres atraviesan, habitan y conviven en la institución objeto de esta investigación.

Uno de los ejes que atravesó los resultados y las discusiones fue la cuestión de las continuidades que signa la práctica docente en el campo de la EF enfocadas en la perspectiva de género. En este punto, vuelve a cobrar la relevancia el pionero trabajo de Saraví (1997) cuando presenta los resultados de una encuesta anónima efectuada a estudiantes de $3^{\circ}$ año en 1996: los discursos del estudiantado presentan puntos en 
común alarmantes en comparación con los enunciados aquí presentados. Entre estas respuesta que datan de 1996 y las actuales de 2020 transcurrieron 24 años; resulta interesante que se repitan las temáticas de los deportes separados por sexo, los enunciados discriminatorios contra las capacidades y posibilidades de las mujeres en general y deportivas en particular, los discursos racistas y de odio contra todo aquello que no esté asociado a la cis-heteronorma, los parámetros hegemónicos de belleza, y los argumentos biologicistas que justifican las desigualdades sociales y educativas.

Hicimos hincapié en la clasificación de una categoría que se repite constantemente entre el material empírico, aquella noción de que la ESI vino a romper lo instituido. Es necesario reflexionar sobre aquello, porque podría ser problemático si es que efectivamente los agentes educativos que construyen la EF escolar tuvieron que esperar más de un siglo desde su institucionalización para que una ley de educación sexual rompa o dispute sus postulados instituyentes. El paradigma pedagógico educativo de la ESI tiene correlatos con parte de los discursos que operan en el campo disciplinar y que tensionan con las perspectivas hegemónicas, problematizando la configuración hegemónica del cuerpo, las desigualdades educativas, las prácticas deportivas competitivas, los roles de género vinculados a las practicas corporales válidas para varones y mujeres, marcos de respeto por el/la otro/a, los discursos militares, médicos, higiénico y morales, entre otras particularidades que constituyen el área de la EF.

Así, la investigación que se presenta en este artículo podría ampliar los horizontes de posibilidad para investigaciones que pretendan problematizar lo corporal, lo deportivo, lo pedagógico, lo histórico, que opera en la formación de la EF en perspectiva de género y de la ESI. Los resultados, discusiones y conclusiones de esta investigación no pecan de conformistas porque "haya algo" de la ESI en la formación docente de EF, sino por el contrario reclama por los derechos que faltan conquistar. De este modo, podemos interpretar a la EF como campo de disputa por la ESI y que la ESI aporta elementos de disputa al interior del campo disciplinar de la EF.

\section{ReFERENCIAS}

Aisenstein, A. y Scharagrodsky, P. (2006) Tras las huellas de la Educación Física Escolar Argentina. Prometeo: Buenos Aires.

Baez, J. (2020) ESI ¿con todas las letras? Un recorrido por los planes de estudio de la formación de profesores/as de Letras en la ciudad de Buenos Aires. Exlibris, 9. Recuperado en: http://revistas.filo.uba.ar/index.php/exlibris/ article/view/3343/2239

Blanco, R. (2014) Estudiantes, militantes, activistas. Nuevas agendas de las agrupaciones universitarias en torno al género y la diversidad sexual. Perfiles Educativos, 36(14). Recuperado en http://www.scielo.org.mx/pdf/peredu /v36n144/v36n144a9.pdf

Butler, J. (2006) Cuerpos que importan. Sobre los límites materiales y discursivos del sexo. Paidós: Bs.As.

Crisorio, R. y Giles, M. (2009) Estudios criticos de Educación Física. Ediciones Al Margen: Buenos Aires.

Crisorio, R. (2015) Actividad(es) física(s) versus prácticas corporales. En Galak, E. y Gambarotta, E. (ed.) Cuerpo, Educación, Politica. Tensiones epistémicas, históricas y prácticas. Editorial Biblos: Buenos Aires

Foucault, M. (2002) Vigilar y Castigar. Nacimiento de la prisión. Buenos Aires: Siglo XXI.

Giachero, F. y Sueiro, R. (2018) Educación Sexual Integral y Formación docente. ¿Qué tiene que ver la educación sexual con mi espacio curricular? Educación, Formación, Investigación, 4(6). Recuperado en: http://ppct.caicyt. gov.ar/index.php/efi/article/view/11360/45454575758737

Kopelovich, P.; Pansa, G. (2017) Educación Fisica, géneros y sexualidades: hacia un estado de la cuestión en Argentina. 12 ${ }^{\circ}$ Congreso Argentino de Educación Física y Ciencias, 13 al 17 de noviembre 2017, Ensenada, Argentina. Educación Física: construyendo nuevos espacios. Ensenada: Universidad Nacional de La Plata. Facultad de Humanidades y Ciencias de la Educación. Departamento de Educación Física. Recuperado en http://www.me moria.fahce.unlp.edu.ar/trab_eventos/ev.10212/ev.10212.pdf 
Marradi, A; Archetti, N; y Piovani, J.I (2007) Metodología de las ciencias sociales. Emecé: Buenos Aires.

Ministerio de Educación de la Nación (2006) Ley Nacional No 26.150. Programa Nacional de Educación Sexual Integral. Boletín Oficial, Buenos Aires.

Ministerio de Educación de la Nación (2008) Lineamientos Curriculares para la Educación Sexual Integral. Programa Nacional de Educación Sexual Integral. Disponible en: http://www.me.gov.ar/me_prog/esi/doc/lineamientos. pdf

Morgade, G. (2015) Hacia una pedagogía sexuada para la justicia. En: Ferreira, A. (comp) Pensando la Educación Física como área de conocimiento. Problematizaciones pedagógicas del sujeto y el cuerpo. Miño y Dávila: Buenos Aires.

Morgade, G.;Fainsod, P.; Del Cerro, C.; y Busca, M. (2016) Educación Sexual con perspectiva de género: reflexiones acerca de su enseñanza en biología y educación para la salud. Biografía, 9(16). Recuperado en: http://www.revi stas.pedagogica.edu.co/index.php/bio-grafia/article/view/4506/3720

Morgade, M; Báez, J; Fainsod, P; Grotz, E. (2018) De omisiones, márgenes y demandas. Las universidades y su papel cardinal en la educación sexual con enfoque de género. En: Rojo, P. y Jardon, V. (comp) Los enfoques de género en las universidades. Recuperado en: http://grupomontevideo.org/ndca/cagenero/wp-content/uploads/2018/ 10/Libro-AUGM-2018.pdf

Savarí, J. (1997) Género y formación docente en educación física. Revista Educación Física y Deporte, 19(2) Recuperado en: https://revistas.udea.edu.co/index.php/educacionfisicaydeporte/article/view/3399/3162

Scharagrodsky, P. (2004) Juntos pero no revueltos: la educación física mixta en clave de género. Cadernos de Pesquisa, 34(121). Recuperado en: https://www.scielo.br/pdf/cp/v34n121/a04n121.pdf

Scharagrodsky, P; Southwell, M. (2007) El cuerpo en la escuela. Buenos Aires: Ministerio de Educación, Ciencia y Tecnología. Disponible en: http://www.memoria.fahce.unlp.edu.ar/libros/pm.869/pm.869.pdf

Sirvent, M.T (2005) El proceso de investigación. EUDEBA: Buenos Aires.

Talani, P. y Viotti, M. (2017) Cuerpos juveniles y educación sexual en la formación docente. Tejiendo relaciones de género(s) equitativas. La aljaba, 21. Recuperado en: https://cerac.unlpam.edu.ar/index.php/aljaba/article/vie $\mathrm{w} / 3482 / 3458$

Tello, C. y Mainardes, J. (2012) La posición epistemológica de los investigadores en Política Educativa: debates teóricos en torno a las perspectivas neomarxista, pluralista y postestructuralista. Archivos Analíticos de Políticas Educativas, 20(9). Recuperado en http://epaa.asu.edu/ojs/article/view/988.

\section{Notas}

1 Entendida ésta como articulación de aspectos biológicos, psicológicos, afectivos, sociales y éticos, superando así la forma de entender la sexualidad meramente desde el punto de vista genital y reproductivo.

2 Sobre este punto, consideramos que sería pertinente realizar un trabajo de investigación aparte que focalice en estas vacantes. A priori, esto podría ser efecto de que la implementación del taller de ESI en los profesorados de EF en la CABA es relativamente reciente. Asimismo, a pesar que se trata de una ley que fue sancionada hace 14 años, aún existen muchos institutos que no la han incluido en su plan de estudios y, por este motivo, no se la toma como objeto de investigación.

3 La inclusión del taller de ESI en el Plan Curricular Institucional (PCI) fue resultado de un doble proceso: por un lado, la modificación del plan de estudio que transitó el instituto durante el 2015; por otro lado, de acuerdo a una normativa regional que estableció la obligatoriedad del taller para los institutos terciarios de formación docente en la CABA.

4 El carácter virtual fue una decisión coyuntural, conforme el aislamiento social preventivo y obligatorio decretado en la República Argentina y en la Ciudad de Buenos Aires durante la elaboración del trabajo de campo de la presente investigación.

5 Véase Scharagrodsky, P. (2007). El cuerpo en la Escuela. Programa de capacitación multimedial. Buenos Aires: Ministerio de Educación, Ciencia y Tecnología.

6 Es necesario remarcar que esta "arbitrariedad docente" estuvo también implicada entre los/as docentes que dictan el taller de ESI. 